\title{
Adipokines as biomarkers of postpartum subclinical endometritis in dairy cows
}

\author{
Gonçalo Pereira ${ }^{1}$, Ricardo Bexiga, João Chagas e Silva', Elisabete Silva', Christelle Ramé2, \\ Joëlle Dupont ${ }^{2}$, Yongzhi Guo ${ }^{3}$, Patrice Humblot ${ }^{3}$ and Luís Lopes-da-Costa ${ }^{1}$ \\ ${ }^{1}$ CIISA - Centro de Investigação Interdisciplinar em Sanidade Animal, Faculdade de Medicina Veterinária, \\ Universidade de Lisboa, Lisboa, Portugal, 'INRAE, UMR85 Physiologie de la Reproduction et des Comportements, \\ Nouzilly, France and ${ }^{3}$ Division of Reproduction, Department of Clinical Sciences, SLU, Uppsala, Sweden \\ Correspondence should be addressed to L Lopes-da-Costa; Email: Icosta@fmv.ulisboa.pt
}

\begin{abstract}
Adipokines emerged as regulators of metabolism and inflammation in several scenarios. This study evaluated the relationship between adipokines (adiponectin, chemerin and visfatin) and cytological (subclinical) endometritis, by comparing healthy (without), transient (recovered by 45 days postpartum (DPP)) and persistent (until 45 DPP) endometritis cows $(n=49)$. Cows with persistent endometritis had higher adiponectin concentrations in plasma (at $21 \mathrm{DPP}, P<0.05$ and at $45 \mathrm{DPP}, \boldsymbol{P}<0.01$ ) and in uterine fluid (at $45 \mathrm{DPP}$, $P<0.001)$, and higher chemerin concentrations in plasma $(P<0.05)$ and uterine fluid $(P<0.01)$ at 45 DPP than healthy cows. Cows with persistent endometritis had higher gene transcription in the cellular pellet of uterine fluid and protein expression in the endometrium of these adipokines and their receptors than healthy cows. Adiponectin plasma concentrations allowed to discriminate healthy from persistent endometritis cows, in 87\% (21 DPP) and 98\% (45 DPP) of cases, and adiponectin and chemerin uterine fluid concentrations at 45 DPP allowed for this discrimination in $\mathbf{1 0 0} \%$ of cases. Cows with concentrations above the cutoff were a minimum of 3.5 (plasma 21 DPP), 20.4 (plasma 45 DPP), and 33.3 (uterine fluid 45 DPP) times more at risk of evidencing persistent endometritis at 45 DPP than cows with concentrations below the cutoff. Overall, results indicate a relationship between adipokine signalling and the inflammatory status of the postpartum uterus of dairy cows, evidencing that adipokines represent suitable biomarkers of subclinical endometritis, able to predict the risk of persistence of inflammation.

Reproduction (2020) 160 417-430
\end{abstract}

\section{Introduction}

Cow fertility is critical for the sustainable worldwide increasing demand of dairy products and the profitability of the dairy industry (Inchaisri et al. 2010, Britt et al. 2018). A main factor impairing fertility is the occurrence of postpartum endometritis, which disrupts ovarian and endometrial function leading to a delay in conception and failure in pregnancy establishment (Mateus et al. 2002, Sheldon et al. 2018). The diagnosis of subclinical endometritis, also termed cytological endometritis, which affects $30-35 \%$ of dairy cows between 4 and 9 weeks postpartum (LeBlanc 2008), remains a challenge (Raliou et al. 2019). Cytological endometritis is an inflammatory state of the endometrium, detected by histology or cytology, in the absence of purulent vaginal discharge and other clinical signs (Sheldon et al. 2006). Due to the invasive nature of the sampling technique, veterinary skills required, time-consuming logistics and cost of the uterine biopsy and swab techniques, the development of reliable, non-invasive biomarkers for the early diagnosis and prognosis of endometritis has been the scope of recent research (Adnane et al. 2017, Mayasari et al. 2017). The early identification of biomarkers that trigger and/or signal the pathological inflammation of the endometrium would enable to predict uterine health status, administer appropriate prophylactic therapy (Adnane et al. 2017) and better manage time of first insemination during the postpartum period.

The adipose tissue serves not only as a depot for lipid storage but also as an endocrine gland that secretes many mediators generally named adipokines (Reverchon et al. 2014), including hormone-like mediators as adiponectin, chemerin and visfatin (Kurowska et al. 2018). Although adipokines are mainly produced by adipocytes and immune cells found in the stromal vascular fraction of adipose tissue, different cell types outside adipose tissue depots have also been described as primary sources of these mediators (Mancuso 2016, Kurowska et al. 2018). Among other functions, adipokines regulate energy metabolism, glucose homeostasis, angiogenesis, reproductive function, immunity and inflammation (Reverchon et al. 2014, Mancuso 2016). 
Mainly produced by white adipose tissue (Reverchon et al. 2014), adiponectin (ADIPOQ) is the most abundant adipokine in human plasma (Barbe et al. 2019). Its primary physiological function is to increase insulin sensitivity, but it is also thought that ADIPOQ plays a major role in suppressing systemic and tissue inflammation due to its anti-inflammatory properties (Fang \& Judd 2018). Its effects are mainly mediated by two seven-transmembrane domain receptors termed ADIPOR1 and ADIPOR2 (Parker-Duffen et al. 2014). These receptors as well as ADIPOQ are expressed in the uterus of humans (Takemura et al. 2006), sows (Smolinska et al. 2014) and cows (Astessiano et al. 2017). In cows, blood ADIPOQ concentrations show a defined pattern, with the nadir around the time of parturition and a progressive increase over the following few weeks of lactation (Barbe et al. 2019), and Kasimanickam et al. (2013) reported increased ADIPOQ serum concentrations in cows with metritis and clinical endometritis compared to heathy cows.

Chemerin, also known as tazarotene-induced gene 2 protein or retinoic acid receptor responder protein 2 (RARRES2), is a proinflammatory adipokine produced by both adipose tissue and liver (Zabel et al. 2014). It is secreted as the inactive precursor prochemerin, which becomes active following cleavage at the C-terminus by extracellular proteases (Mattern et al. 2014) and contributes to the regulation of adipogenesis, insulin secretion and the inflammatory process (Kurowska et al. 2018). In the latter, RARRES2 works as a chemoattractant for monocytes and dendritic cells (Mancuso 2016), and its blood concentrations correlate with those of TNF $\alpha$, IL-6 and C-reactive protein (Rourke et al. 2013). Chemerin binds to three seven-transmembrane domain receptors - CMKLR1 (Chemokine like receptor 1), CCRL2 (C-C chemokine receptor-like 2) and GPR1 (G Protein-Coupled Receptor 1) (Mariani \& Roncucci 2015). The activation of CMKLR1 by RARRES2 induces the migration of macrophages and dendritic cells in vitro, which supports the proinflammatory role of the RARRES2/CMKLR1 axis (Yoshimura \& Oppenheim 2011). In cows, RARRES2 was referred as a mediator, linking metabolism and ovarian function, and plasma concentrations are positively correlated with body fat mobilization and milk yield (Mellouk et al. 2019).

Visfatin, also known as pre-B-cell colony enhancing factor or nicotinamide phosphoribosyl-transferase (NAMPT), is predominantly expressed in visceral adipose tissue but also in muscle, bone marrow, liver, lymphocytes and foetal membranes (Reverchon et al. 2014, Dupont et al. 2015). Visfatin has an immunomodulatory function and is involved in obesity, inflammation and insulin resistance (Zhang et al. 2019). In cows, peripartal serum concentrations of NAMPT were proposed as predictive indicators of retained placenta and other inflammatory diseases (Fadden \& Bobe 2016).
Puerperal dairy cows are under metabolic stress, which is related to uterine inflammation (Hammon et al. 2006, Guo et al. 2019). Owing to the regulation of metabolism and inflammation by adipokines, these molecules may represent promising biomarkers for the early diagnosis and prognosis of postpartum subclinical endometritis. The objective of this study was to evaluate the relationship between adipokines (adiponectin, chemerin, visfatin and their receptors) and postpartum endometritis in dairy cows. The hypothesis behind this study was that adipokine signalling may be active in the cow uterus and that this signalling pattern and adipokine concentrations (blood and uterine) may be related to the inflammatory status of the uterus.

\section{Materials and methods}

\section{Ethics statement}

The experiments were conducted in compliance with the Portuguese legislation for the use of animals for experimental purposes (Decreto-Lei $n^{\circ} 129 / 92$ and Portaria $n^{\circ} 1005 / 92$, DR $n^{\circ} 245$, série I-B, 4930-42) and with the European Union legislation (Directive 2010/63/UE). The research protocol was approved by the Institutional Animal Care and Use Committee (Reference CEIE n³7/2019). All animal technical procedures were performed by licenced veterinarians, all of which are accredited as FELASA category C scientists or equivalent.

\section{Animals, sampling and experimental design}

Sample collection was performed in a 400 milking HolsteinFriesian dairy cow herd, with a three daily milking routine and a milk yield average of 11,500 L/cow/305 days of lactation. After calving, cows were monitored daily for signs of puerperal disease. Cows that experienced dystocia, retained foetal membranes, puerperal metritis or mastitis, clinical hypocalcaemia, ketosis and lameness were not included in the study, and enrolled cows did not receive antibiotic or antiinflammatory therapy throughout the study. Selected cows $(n=49)$ were examined and sampled at $21(21 \pm 0.4)$ and 45 $(44 \pm 0.7)$ days postpartum (DPP). At 21 DPP, cows were blood sampled, the score of the vaginal discharge was assessed, the genital tract was evaluated through transrectal palpation and ultrasonography, and an endometrial swab was taken for cytological examination. At 45 DPP the same procedures were repeated. In addition, as described in details subsequently, a small volume flush and an endometrial biopsy were collected from both uterine horns. Once the voluntary waiting period (50 DPP) was over, cows were submitted to artificial insemination (AI) at the ensuing estrus (detected by visual observation plus pedometer measured walking activity). At the entrance of the milking parlour, an electronic collar identified cows and milk yield and body weight were automatically recorded after each milking. Data including milk yield and body weight (three times daily), estrus and insemination dates, and pregnancy status (at 35-42 and 90 days post-Al) were retrieved from the herd management software, until completion of lactation (305 DPP). 
The experimental design considered the comparison between three groups, with cows being retrospectively allocated to: (1) control healthy cows, without cytological endometritis at 21 and 45 DPP (group $\mathrm{HH} ; n=19$ ); (2) cows with cytological endometritis at 21 DPP that had recovered by 45 DPP (group $\mathrm{EH} ; n=19$ ); and (3) cows with cytological endometritis at 21 DPP, which persisted at 45 DPP (group EE; $n=11$ ). A further refinement of group $\mathrm{HH}$ considered two subsets of cows, including cows pregnant at first $\mathrm{Al}$ (subset HHP; $n=6$ ) and cows pregnant at subsequent Als $(n=13)$. The subset HHP was considered for 'extreme cases' comparison (healthy pregnant vs endometritis) in endometrial immunolocalization and transcription analysis of the cellular pellet of uterine flushing.

\section{Blood sampling}

Blood samples were aseptically collected by venipuncture of the coccygeal vein into $10 \mathrm{~mL}$ dry tubes and $10 \mathrm{~mL}$ tubes containing K3 EDTA (13060, Vacutest KIMA, Arzegrande, Italy). Tubes were immediately centrifuged (2000 $\mathrm{g}$ for $15 \mathrm{~min}$ ), plasma and serum were aliquoted into $1.5 \mathrm{~mL}$ Eppendorfs and stored at $-20^{\circ} \mathrm{C}$ until subsequent analysis for adipokines, nonesterified fatty acids (NEFA) and progesterone concentrations.

\section{Genital tract evaluation}

The genital tract was palpated per-rectum to assess the size of the cervix and uterus and the symmetry and tonus of uterine horns. An ensuing ultrasound examination (ExaGo, Echo Control Medical, France) evaluated the uterine content (intrauterine fluid volume and echogenicity) and identified and measured the ovarian structures. The vaginal discharge score was graded in a 0-3 scale according to Williams et al. (2005), following collection with a Metricheck device (EndoControl Sampler, Minitube, La Selva del Camp, Spain).

\section{Endometrial cytology}

Endometrial swabs were performed using an adapted cytobrush technique. A cervical brush (Bastos Viegas SA, Penafiel, Portugal) was aseptically adapted to the inner stylet of and retracted into an $\mathrm{Al}$ gun (IMV technologies, L'Aigle, France) covered with an $\mathrm{Al}$ sheath (IMV technologies), and the swab obtained as described by Pascottini et al. (2016). The brush was then gently rolled along the length of two glass microscope slides, which were subsequently labelled and air-dried. Slides were stained with a modified Wright-Giemsa ${ }^{\circledR}$ stain (Diff-Quick, MAIM SL, Barcelona, Spain) and the percentage of polymorphonuclear neutrophils (PMN) was assessed from 400 cells (200 in each slide). At 21 DPP a $\geq 18 \%$ PMN cut-off was chosen (Kasimanickam et al. 2004), and cows with a PMN percentage below this cutoff were considered without cytological endometritis. At 45 DPP, $a \geq 5 \%$ PMN cut-off was chosen (Gilbert et al. 2005) and, similarly, cows with a PMN percentage below this cut-off were considered without cytological endometritis.

\section{Uterine flushing}

Both uterine horns were flushed independently with sterile PBS (Millipore Corp), using a sterile silicone-coated latex Foley catheter (Rusch Gold, Rusch, Perak, Malaysia) aseptically mounted in a metal stylet and covered with a sanitary sheath (IMV technologies). At the cervical external opening, the sanitary sheath was retracted, the catheter guided to one of the uterine horns past the external bifurcation level, and the cuff inflated. Fifty milliliters of PBS were infused into each uterine horn, which was gently massaged per-rectum, before allowing collection of the fluid into a $50 \mathrm{~mL}$ centrifuge tube. Samples were transported to the laboratory at $4{ }^{\circ} \mathrm{C}$, filtered with a mesh to remove mucus and debris, centrifuged (5000 $\mathbf{g}$ for $15 \mathrm{~min}$ ), and the supernatant and cellular pellet independently stored at $-80^{\circ} \mathrm{C}$ until assayed, respectively, for adipokines' concentrations and transcription of adipokine signalling genes.

\section{Endometrial biopsy}

Endometrial biopsies were independently collected from both uterine horns by using Kervokian-Younge endometrial biopsy instrument (Alcyon, Paris, France), according to procedures described by Chapwanya et al. (2009, 2010). Briefly, a low epidural anaesthesia was performed with $200 \mathrm{mg}$ of Procaine hydrochloride $+0.18 \mathrm{mg}$ of Epinephrine tartrate (Pronestesic, Fatro, Bologna, Italy) injected in the epidural cavity at the first coccygeal space. After hygiene of the vulva, the sterile biopsy instrument, guarded in a protective sheath, was introduced into the vagina and advanced to the external cervical orifice, where the protective sheath was ruptured. The biopsy instrument was then guided into the first third of the uterine horn and an endometrial sample of about $1.5 \mathrm{~cm}^{2}$ was recovered. The endometrial sample was cut into three equal size portions, one of which was immediately fixed in $4 \%$ paraformaldehyde (PFA) and paraffin embedded within 24-48 $\mathrm{h}$ for immunohistochemistry analysis. The remaining portions were processed for other analysis.

\section{Progesterone assay}

Serum progesterone was assayed by a chemiluminescent immunoassay in an IMMULITE 1000 analyser (Siemens Healthcare Diagnostics), using a commercial kit (IMMULITE 1000 Progesterone Kit, Siemens Healthcare Diagnostics). The analytical sensitivity of the assay was $0.2 \mathrm{ng} / \mathrm{mL}$ and the interassay coefficient of variation was $<10 \%$. Cows were classified as in luteal phase (at 21 and 45 DPP) when progesterone concentration was $>0.5 \mathrm{ng} / \mathrm{mL}$ and an ovarian luteal structure (corpus luteum) was detected during the ultrasound examination.

\section{Non-esterified fatty acids assay}

Serum NEFA concentrations were determined by a colorimetric method (kit no. FA 115, Randox, Crumlin, UK) using a Randox RX Daytona equipment (Randox). Each sample was analysed in duplicate and the quality control of the assay was performed with the Randox Acusera Assayed Chemistry Premium Plus Control (Assayed Chemistry Premium Plus Level 2 and 3, Randox). The analytical sensitivity of the assay was 0.072 $\mathrm{mmol} / \mathrm{L}$ and the inter-assay coefficient of variation was $<5 \%$. 


\section{Adipokine assays}

Plasma and uterine fluid total ADIPOQ, RARRES2 and NAMPT concentrations were measured by using ELISA kits (E11A0125 and E11C0104 from BlueGene, Shanghai, China and EK-00380 from Phoenix France, SAS, Strasbourg, France, respectively), as previously described (Fadden \& Bobe 2016, Mellouk et al. $2017,2019)$. The intra-assay coefficients of variation for total ADIPOQ, RARRES2 and NAMPT were $6 \%, 4.5 \%$ and $6.5 \%$, respectively. The inter-assay coefficients of variation for total ADIPOQ, RARRES2 and NAMPT were $6.2 \%, 6.5 \%$ and $6 \%$, respectively. Adipokine uterine fluid measurements (as well as quantitative real time RT-PCR of the uterine fluid cell pellet; see subsequently) were performed in samples recovered from the uterine horn contralateral to the corpus luteum or to a randomly selected uterine horn if no corpus luteum was present.

\section{Immunohistochemistry}

Endometrial samples from three cows of each group (HHP, EH and EE) were used for protein immunolocalisation. Paraffin embedded endometrial samples were serially sectioned at a thickness of $4 \mu \mathrm{m}$, deparaffinised and hydrated. Quenching of endogenous peroxidase activity, antigen retrieval (EnVision ${ }^{\mathrm{TM}}$ FLEX Target Retrieval Solution, DM829; Dako, Glostrup, Denmark) and nonspecific background elimination were performed according to Maillard et al. (2010). Twin sections were incubated overnight at $4^{\circ} \mathrm{C}$ with control rabbit or mouse $\lg \mathrm{G}$ and the different primary antibodies (Supplementary Table 1 , see section on supplementary materials given at the end of this article) diluted in PBS with 5\% lamb serum. Sections were then washed four times in PBS for $10 \mathrm{~min}$ and incubated for 30 $\mathrm{min}$ at room temperature with a ready-to-use labelled polymerHRP (EnVision ${ }^{\text {TM }}$ FLEX/HRP detection reagent, SM802; Dako). After washing twice in PBS for 10 min, immunoreactivity was revealed by incubation with DAB chromagen/DAB buffer at room temperature, according to manufacturer instructions (Envision ${ }^{\mathrm{TM}}$ FLEX, K8000; Dako). Finally, the slides were counterstained with Meyers haematoxylin (Merck), then dehydrated and mounted with Entellan ${ }^{\circledR}$ (Merck). Staining intensity and cell type identification were independently assessed by two observers, at $100 \times$ and $1000 \times$ magnification. Intensity was classified as (absent); +/- (weak); +(moderate); ++ (strong). From each cow, staining was assessed from a minimum of three optical fields from twin slides for each antibody.

\section{Quantitative real time RT-PCR analysis of ADIPOQ, ADIPOR1, ADIPOR2, RARRES2, CMKLR1 and GPR1 in the cellular pellet of the uterine flushing}

Total RNA of the cellular pellet of the uterine flushing performed at 45 DPP was extracted using the RNeasy Midi kit (Qiagen $(\mathbb{)})$, according to manufacturer instructions. Concentration and purity of RNA were determined with a NanoDrop Spectrophotometer (Peqlab Biotechnologie GmbH, Erlangen, Germany), RNA integrity evaluated on $1.25 \%$ agarose-formaldehyde gels, and total RNA quality assessed with an Agilent Bioanalyzer 2100, using a RNA 6000 pico kit (Agilent Technologies). The 260/280 ratio (1.7-1.9) and RIN value (7.2-8.5) evidenced a suitable RNA quality for analysis (Supplementary Table 2). The cDNA was generated by RT of total RNA $(1 \mu \mathrm{g})$ in a mixture comprising $0.5 \mathrm{mM}$ of each deoxyribonucleotide triphosphate (dATP, dGTP, dCTP, DTTP), $2 \mathrm{M}$ of RT buffer, $15 \mu \mathrm{g} / \mu \mathrm{L}$ of oligodT, $0.125 \mathrm{U}$ of ribonuclease inhibitor, and 0.05 UMMLV (Moloney murine leukemia virus reverse transcriptase) for 1 hour at $37^{\circ} \mathrm{C}$. Real-time PCR was performed using the MyiQ Cycle device (Bio-Rad), in a mixture of SYBR Green Supermix 1X reagent (Bio-Rad), $250 \mathrm{nM}$ specific primers (Invitrogen ${ }^{\mathrm{TM}}$ by Life Technologies ${ }^{\mathrm{TM}}$ ) (Supplementary Table 3 ) and $5 \mu \mathrm{L}$ of cDNA diluted $1: 5$, in a total volume of $20 \mu \mathrm{L}$. The amplification protocol started with an incubation for $2 \mathrm{~min}$ at $50^{\circ} \mathrm{C}$ and a denaturation step of $10 \mathrm{~min}$ at $95^{\circ} \mathrm{C}$, followed by 40 cycles $\left(30 \mathrm{~s}\right.$ at $95^{\circ} \mathrm{C}, 30 \mathrm{~s}$ at $60^{\circ} \mathrm{C}, 30 \mathrm{~s}$ at $72^{\circ} \mathrm{C}$ ), and acquisition of the melting curve. Each sample was analysed in duplicate in the same plate, and amplification with water, instead of cDNA, was performed systematically as a negative control. Gene transcription was calculated according to primer efficiency $(\mathrm{E})$ and quantification cycle $(\mathrm{Cq})$, where transcription $n=\mathrm{E}-\mathrm{Cq}$. Then, relative expression of the target gene was analyzed following standardization of the level of mRNA expression for the geometric mean of three reference genes (GAPDH, ACTB and PPIA) which were reported as accurate normalisation factors (Vandesompele et al. 2002).

\section{Statistical analysis}

Data were managed in Microsoft Excel and statistical analyses were conducted using SPSS for Windows 26.0 (IBM Corp.). The normality of the distribution of the data was tested with the Shapiro-Wilk-Test. Values are reported as median and interquartile range for non-normally distributed variables and mean \pm S.E.M. for those normally distributed (Supplementary Table 4). Significant differences between groups were determined using a one-way ANOVA or the non-parametric Kruskal-Wallis-Test with Dunns post-test and Bonferroni correction for multiple tests, respectively, for normally and non-normally distributed variables. The level of significance was set at $P<0.05$. Correlation analysis used the Spearman's rank test. The predictive accuracy of the different adipokines to discriminate cows with cytological endometritis was evaluated by Receiver Operating Characteristic (ROC) curve analysis, testing the probability $(P)$ that the area under curve (AUC) differed significantly from random $(A \cup C=0.5)$. ROC curve coordinates allowed Youden Index calculation, best cut-off and odds ratio (OR) determination for ADIPOQ and RARRES2 concentrations. Cohen's kappa statistic was used to assess the agreement between the vaginal discharge Metricheck score and the endometrial cytology PMN percentage.

\section{Results}

\section{Endometrial cytology PMN percentage and vaginal discharge Metricheck score}

Endometrial cytology PMN percentage reflects the retrospective allocation of cows to groups, based on endometrial cytology cut-off (Table 1). At 21 DPP, the endometrial cytology PMN percentage was lower 
in group $\mathrm{HH}$ than in groups $\mathrm{EH}$ and $\mathrm{EE}(P<0.001)$. However, at 45 DPP, the endometrial cytology PMN percentage was similar in groups $\mathrm{HH}$ and $\mathrm{EH}$ and lower than in group EE $(P<0.001)$. At $21 \mathrm{DPP}$, the vaginal discharge Metricheck score was lower $(P=0.001)$ in group $\mathrm{HH}$ than in group $\mathrm{EE}$, but at $45 \mathrm{DPP}$, the vaginal discharge Metricheck score was similar in all groups. At 21 DPP, there was a moderate level of agreement (Cohen's Kappa $=0.47 ; P<0.01$ ) between the vaginal discharge Metricheck score and the endometrial cytology PMN percentage (Supplementary Table 5A). However, at 45 DPP, there was no agreement between the mentioned parameters (Cohen's Kappa $=-0.10$; $P=0.46)$ (Supplementary Table 5B).

\section{Metabolic and fertility parameters}

As shown in Table 1, at 21 DPP and 45 DPP, there were no significant differences between groups in mean milk yield, body weight loss and serum NEFA concentrations. There were no significant differences for the calving to first $\mathrm{Al}$ interval, calving to conception interval and Al number/conception between the three groups. However, the latter two parameters of groups $\mathrm{EH}$ and $\mathrm{EE}$ were favoured by only including pregnant cows within 305 DPP (as five and four cows were culled for nonconception at 305 DPP, respectively). Six of 19 (32\%) $\mathrm{HH}$ cows conceived at first $\mathrm{Al}$ and the remaining were pregnant within 305 DPP. Also, at 45 DPP there were more cows in luteal phase in group $\mathrm{HH}$ than in groups $\mathrm{EH}$ and $\mathrm{EE}(P=0.01)$, evidencing an earlier resumption of ovarian activity of $\mathrm{HH}$ cows, compared to $\mathrm{EH}$ and $\mathrm{EE}$ cows.

\section{Adipokine concentrations in plasma and uterine fluid}

Plasma and uterine fluid concentrations of ADIPOQ, RARRES2 and NAMPT are presented in Fig. 1 and Supplementary Table 6. ADIPOQ concentrations in plasma at $21 \quad(P<0.05)$ and $45 \operatorname{DPP}(P<0.01)$ and uterine fluid $(P<0.001)$ were higher in EE cows than in $\mathrm{HH}$ and $\mathrm{EH}$ cows. At $45 \mathrm{DPP}$, plasma concentrations of RARRES2 were higher $(P<0.05)$ in $\mathrm{EE}$ than in $\mathrm{HH}$ cows and uterine fluid concentrations were higher $(P<0.01)$ in group $\mathrm{EE}$ than in groups $\mathrm{HH}$ and $\mathrm{EH}$. In contrast, uterine fluid concentrations of NAMPT were similar in the three groups. Nevertheless, plasma NAMPT concentrations of EH cows were lower $(P<0.01)$ than of EE cows at 21 DPP and of both $\mathrm{HH}$ and EE cows at $45 \mathrm{DPP}(P<0.01)$. Plasma concentrations of ADIPOQ, RARRES2 and NAMPT at 21 DPP and 45 DPP were similar in luteal phase and follicular/anoestrous cows of groups $\mathrm{HH}, \mathrm{EH}$ and $\mathrm{EE}$. This was also observed in uterine concentrations at 45 DPP, except in $\mathrm{HH}$ group, where only ADIPOQ concentrations were higher $(P<0.01)$ in luteal than in follicular/anoestrous cows (data not shown).

Table 1 Metabolic and fertility parameters in healthy cows (group $\mathrm{HH}$ ), cows with cytological endometritis at 21 DPP but that recovered by 45 DPP (group EH), and cows with persistent cytological endometritis until 45 DPP (group EE).

\begin{tabular}{|c|c|c|c|}
\hline Parameters & HH $(n=19)$ & EH $(n=19)$ & $\mathbf{E E}(n=11)$ \\
\hline Lactation number* & $1(1-2)$ & $2(1-2)$ & $2(1-3)$ \\
\hline Body Weight Loss Calving - 21 DPP (\%)* & $2.2(0-5.4)$ & $1.9(-1.2-5.8)$ & $7.5(-0.2-9.0)$ \\
\hline Body Weight Loss Calving - 45 DPP (\%)* & $4.1 \pm 1.8$ & $2.5 \pm 1.4$ & $3.9 \pm 1.8$ \\
\hline Body Weight Loss Calving - 60 DPP (\%)* & $3.6(1.6-7.8)$ & $1.3(-1.9-5.0)$ & $4.9(-1.1-10.2)$ \\
\hline Milk yield by 21 DPP $(\mathrm{L})^{* *}$ & $863.5 \pm 38.7$ & $839.8 \pm 33.0$ & $874.0 \pm 48.6$ \\
\hline Milk yield by 45 DPP $(\mathrm{L})^{* *}$ & $1716.8 \pm 76.5$ & $1710.0 \pm 64.7$ & $1806.7 \pm 95.0$ \\
\hline Milk yield by 60 DPP $(\mathrm{L})^{* *}$ & $2375.2 \pm 102.1$ & $2392.6 \pm 88.0$ & $2512.4 \pm 133.7$ \\
\hline $\mathrm{NEFA}$ at $21 \mathrm{DPP}(\mathrm{mmol} / \mathrm{L})^{*}$ & $0.28(0.17-0.50)$ & $0.25(0.17-0.60)$ & $0.26(0.20-0.43)$ \\
\hline NEFA at 45 DPP $(\mathrm{mmol} / \mathrm{L})^{*}$ & $0.27(0.17-0.50)$ & $0.28(0.14-0.43)$ & $0.17(0.13-0.34)$ \\
\hline Cows in luteal phase at 21 DPP $(n(\%))$ & $14(74)^{\mathrm{a}}$ & $5(26)^{b}$ & $6(55)^{a, b}$ \\
\hline Cows in luteal phase at $45 \operatorname{DPP}(n(\%))$ & $18(89)^{\mathrm{a}}$ & $10(53)^{\mathrm{b}}$ & $7(64)^{b}$ \\
\hline Metricheck Score at 21 DPP* & $0(0-1)^{\mathrm{a}}$ & $1(0-2)^{a, b}$ & $2(2-2)^{b}$ \\
\hline Metricheck Score at 45 DPP* & $0(0-0)$ & $0(0-0)$ & $0(0-0)$ \\
\hline PMN \% in Cytobrush at 21 DPP* & $4.0(1.0-8.0)^{\mathrm{a}}$ & $25.5(20-41.5)^{b}$ & $61(35-86)^{b}$ \\
\hline PMN \% in Cytobrush at 45 DPP* & $1(0.0-1.0)^{\mathrm{a}}$ & $1(0-1)^{\mathrm{a}}$ & $11(7-17)^{b}$ \\
\hline Calving - first $\mathrm{Al}$ interval (days)* & $61(56-67)$ & $61(58-80)$ & $64(50-94)$ \\
\hline Cows pregnant at first $\mathrm{Al}(n(\%))$ & $6(32)$ & 0 & 0 \\
\hline Cows conceiving within 305 DPP $(n(\%))$ & $19(100)$ & $14(74)$ & $7(64)$ \\
\hline Calving - conception interval (days)* & $136(64-232)$ & $110(98-157)$ & $94(85-236)$ \\
\hline$n$ & 19 & 14 & 7 \\
\hline Al number/conception* & $4(1-6)$ & $3(2-4)$ & $2(2-7)$ \\
\hline$n$ & 19 & 14 & 7 \\
\hline
\end{tabular}

Different letters indicate significant differences between groups (level of significance $P<0.05$ ) determined with ANOVA and the non-parametric Kruskal-Wallis test with Dunns post-test for normally and non-normally distributed variables, respectively.

*Values reported as median and (interquartile range) for non-normally distributed data; **Values reported as mean \pm S.E.M. for normally distributed data.

DPP, days postpartum. 

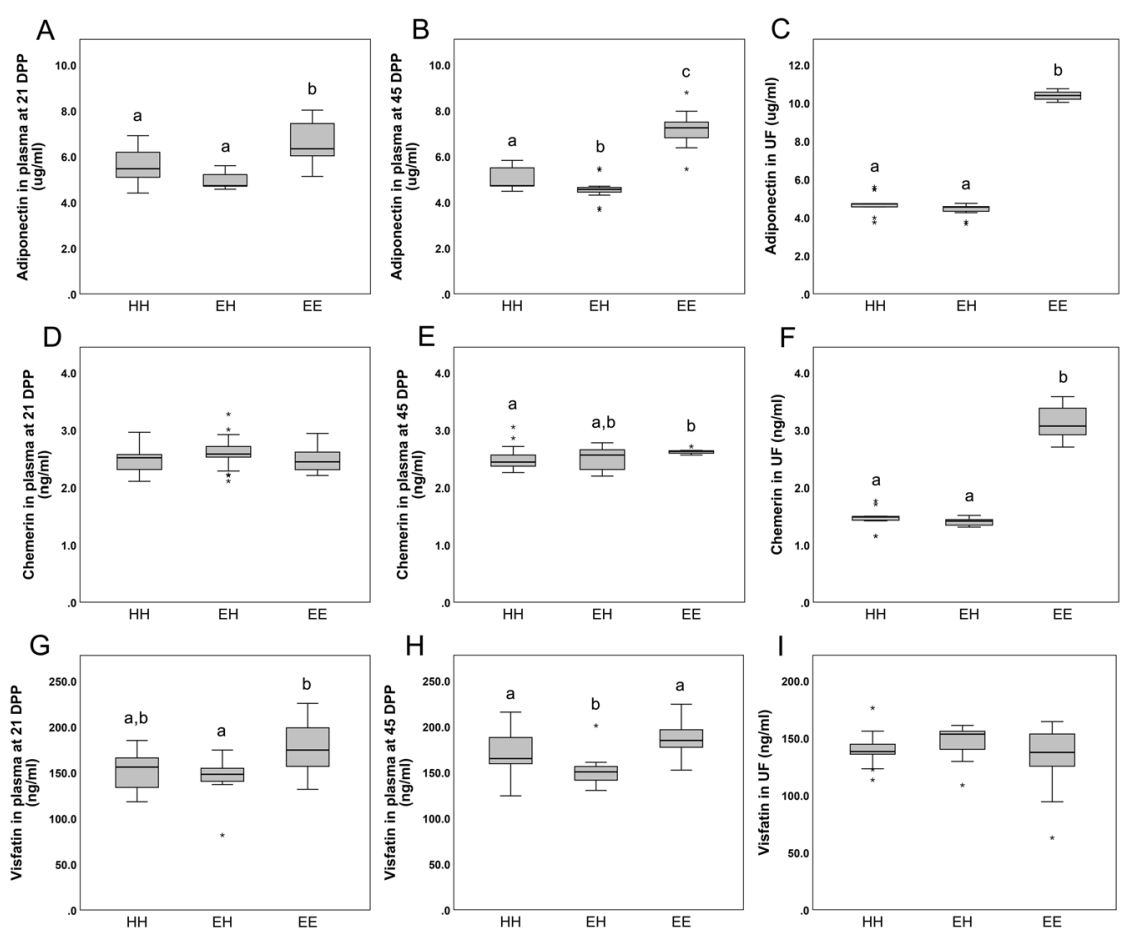

Figure 1 Plasma and uterine fluid concentrations of adiponectin (A, B and C), chemerin (D, E and F) and visfatin $(\mathrm{G}, \mathrm{H}$ and $\mathrm{I})$ in healthy cows (group $\mathrm{HH}$ ), cows with cytological endometritis at 21 DPP but recovered by 45 DPP (group EH), and cows with persistent cytological endometritis until 45 DPP (group EE). Different letters indicate a significant difference at $P<0.05$. Horizontal black lines indicate median, boxes extend from the 25th to the 75th percentile and vertical lines indicate values within 1.5 interquartile range of the 25 th and 75 th percentile. Asterisks indicate outliers.

\section{Relationship between adipokines' concentrations and PMN percentage from endometrial cytology}

Table 2 displays a summary of Spearman correlations between adipokines' concentrations and PMN percentage from endometrial cytology. Uterine fluid concentrations of ADIPOQ and RARRES2 showed a significant positive correlation with the endometrial cytology PMN percentage at 45 DPP. In addition, concentrations of ADIPOQ in plasma significantly correlated with those in uterine fluid and at 45 DPP with the endometrial cytology PMN percentage. RARRES2 uterine fluid levels did not correlate with its plasma levels at 45 DPP.

Figure 2 represents the ROC analysis of ADIPOQ and RARRES2 concentrations, to discriminate cows with cytological endometritis at 45 DPP. Plasma ADIPOQ at $21 \mathrm{DPP}(\mathrm{AUC}=0.872 ; P<0.001$ ), plasma ADIPOQ at 45 DPP (AUC $=0.981 ; P<0.001)$, and uterine fluid ADIPOQ and RARRES2 at 45 DPP $(A \cup C=1.000$;
$P<0.001)$ showed high AUC values. In contrast, plasma RARRES2 at 21 DPP (AUC $=0.423 ; P=0.443$ ) and at 45 DPP (AUC $=0.738 ; P=0.017$ ) showed either no ability or a low ability to discriminate cows with cytological endometritis. The risk of endometritis persistence at 45 DPP (Supplementary Table 7) was higher for cows with plasma ADIPOQ concentrations at 21 DPP above 5.9 $\mu \mathrm{g} / \mathrm{mL}(\mathrm{OR}=19.9 ; 95 \% \mathrm{Cl}: 3.5-113.3 ; P<0.001)$ than for cows with concentrations below the cutoff. At 45 DPP, plasma and uterine fluid ADIPOQ concentrations showed a high discriminatory power for diagnosis of endometritis. Cows with plasma and uterine fluid ADIPOQ concentrations above 6.1 and $7.8 \mu \mathrm{g} / \mathrm{mL}$, respectively, had increased risk of being diagnosed with endometritis $(\mathrm{OR}=539 ;$; $95 \mathrm{Cl}$ : 20.4-14,218.7; $P<0.001$ and $\mathrm{OR}=1771 ; 95 \% \mathrm{Cl}: 33.3-94,301.3$; $P<0.001$, respectively) than cows with concentrations below the cutoff. For plasma concentrations, only 1 of 39 cows with concentrations below the cutoff showed

Table 2 Spearman's correlations between the plasma (21 and 45 DPP) and uterine fluid (UF; 45 DPP) adipokines' concentrations and the PMN percentage from endometrial cytology $(n=49)$.

\begin{tabular}{|c|c|c|c|c|c|c|c|}
\hline & $\begin{array}{c}\text { ADIPOQ } \\
21 \text { DPP }\end{array}$ & $\begin{array}{c}\text { ADIPOQ } \\
45 \text { DPP }\end{array}$ & ADIPOQ UF & $\begin{array}{c}\text { RARRES2 } \\
45 \text { DPP }\end{array}$ & RARRES2 UF & $\begin{array}{l}\text { PMN \% } \\
21 \text { DPP }\end{array}$ & $\begin{array}{l}\text { PMN \% } \\
45 \text { DPP }\end{array}$ \\
\hline ADIPOQ 21 DPP & & $0.599 * *$ & $0.631 * *$ & $0.349 *$ & $0.457^{* *}$ & $0.087^{\mathrm{ns}}$ & $0.281^{\mathrm{ns}}$ \\
\hline ADIPOQ 45 DPP & & & $0.704^{* *}$ & $0.216^{\mathrm{ns}}$ & $0.720^{* *}$ & $0.087^{\mathrm{ns}}$ & $0.436^{* *}$ \\
\hline ADIPOQ UF & & & & $0.093^{\mathrm{ns}}$ & $0.782^{* *}$ & $0.244^{\mathrm{ns}}$ & $0.537^{* *}$ \\
\hline RARRES2 45 DPP & & & & & $-0.019^{\mathrm{ns}}$ & $0.231^{\mathrm{ns}}$ & $0.153^{\text {ns }}$ \\
\hline RARRES2 UF & & & & & & $0.218^{\mathrm{ns}}$ & $0.640^{* *}$ \\
\hline PMN \% & & & & & & & $0.507^{* *}$ \\
\hline
\end{tabular}

$* P<0.05$ and ${ }^{*} * P<0.01$.

DPP, days postpartum; ${ }^{\text {ns }}$, not significant. 

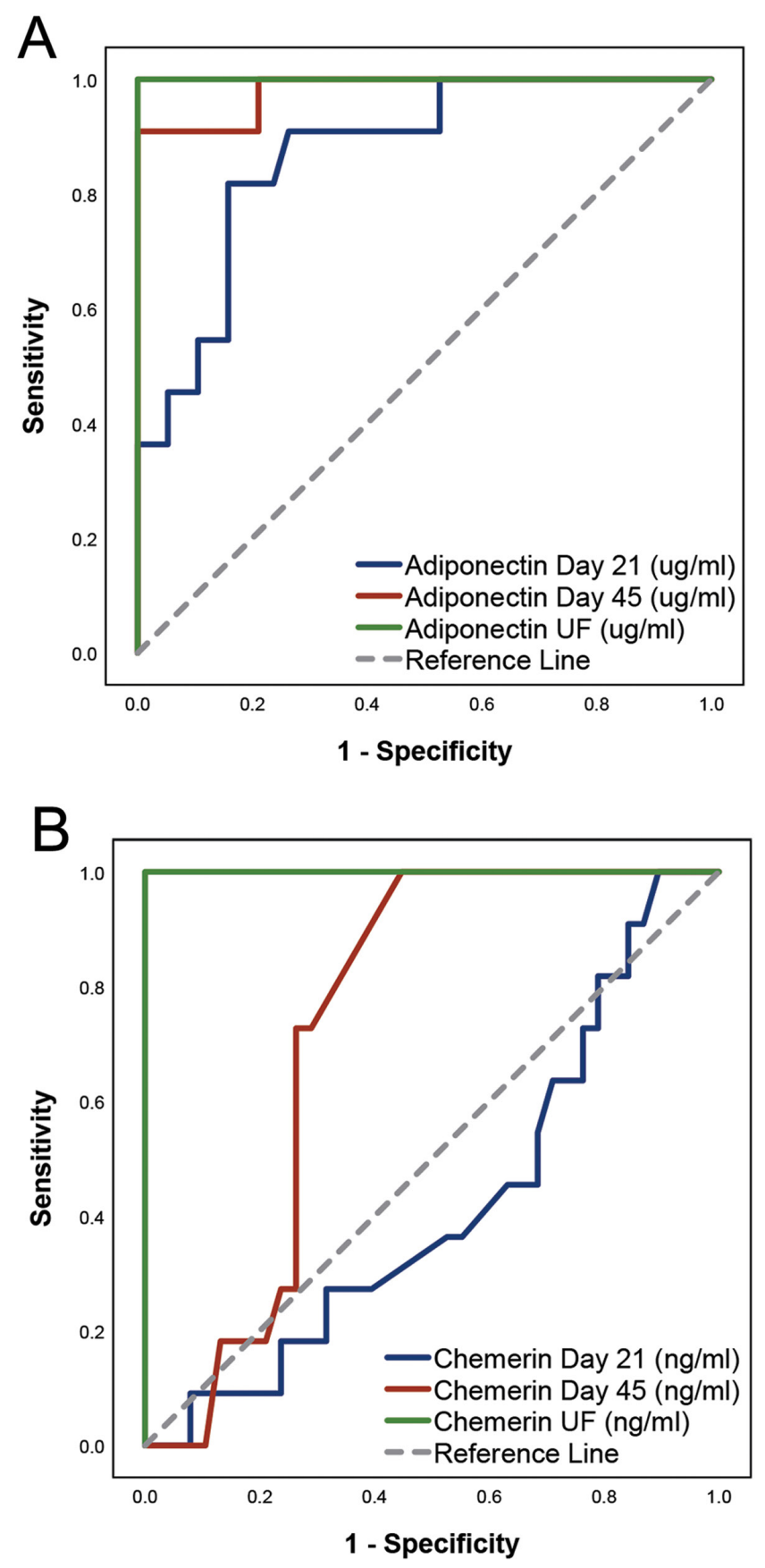

Figure 2 Receiver operating characteristic curve analysis of adiponectin (A) and chemerin (B) to discriminate cows with cytological endometritis at 45 days postpartum. Concentrations were measured in plasma at 21 and 45 days postpartum and in uterine fluid (UF) at 45 days postpartum.

endometritis and all 10 cows with concentrations above the cutoff presented endometritis. For uterine fluid concentrations, all cows with concentrations below the cutoff were healthy, whereas all cows with concentrations above the cutoff presented endometritis. This was also observed in the case of uterine fluid RARRES2 concentrations (cutoff $=2.2 \mathrm{ng} / \mathrm{mL}$ ).
As concentrations in plasma and uterine fluid showed significant differences between healthy $(\mathrm{HH})$ and endometritis cows (EE) only for ADIPOQ and RARRES2, the analysis of the endometrial immunolocalisation and of the gene expression in cellular pellets from uterine flushings was performed only for these two adipokines and their receptors. Also, to further refine the evaluation of differences between healthy and endometritis cows ('extreme cases'), the analysis considered a subset of the $\mathrm{HH}$ group, consisting of healthy cows that became pregnant at first $\mathrm{Al}$ (group HHP; $n=6$ ).

\section{Immunolocalisation of ADIPOQ, ADIPOR1, ADIPOR2, RARRES2 and CMKLR1 in the bovine endometrium}

Positive immunostaining for ADIPOQ was observed in the luminal and glandular epithelial cells and in endothelial, stromal and inflammatory cells of all cows. However, cows of group EE exhibited a stronger staining in the luminal epithelium than cows of HHP and $\mathrm{EH}$ groups (Fig. 3A, B, C and Table 3). Positive staining for ADIPOQ was observed in endothelial cells (Fig. 3D) and macrophages (Fig. 3F) within the stroma, whereas the infiltrated $\mathrm{PMN}$ were negative (Fig. 3E). Positive immunostaining for ADIPOR1 and ADIPOR2 was observed in luminal and glandular epithelial cells. Additionally, ADIPOR2 staining was observed in stromal and inflammatory cells (Fig. 3G, H, I, J, K and $\mathrm{L}$ ). Both these receptors displayed a stronger staining in EE cows than in HHP and EH cows (Table 3). Positive immunostaining for RARRES2 was detected in the luminal and glandular epithelia and in stroma and inflammatory cells of all cows (Fig. 4A, B and C), and the level of staining was stronger in EE group than in $\mathrm{HHP}$ and EH groups (Table 3). Positive immunostaining for CMKLR1 was observed in the luminal epithelium and in stroma and inflammatory cells (Fig. 4D, E and F). However, in the luminal epithelium of HHP and $\mathrm{EH}$ cows, the staining was mostly found in the apical membrane of the cells (Fig. 4D and E), whereas in EE cows a strong staining is present throughout the cytoplasm (Fig. 4F).

\section{Transcription levels of ADIPOQ, ADIPOR1, ADIPOR2, RARRES2, CMKLR1, GPR1 and CCRL2 in the cellular pellet of the uterine flushing}

Transcription levels of ADIPOQ, ADIPOR1, ADIPOR2, RARRES2, CMKLR1 and GPR1 are presented in Figs 5 and 6. Transcription levels of $A D I P O Q$ and ADIPOR2 were higher $(P<0.001)$ in group $\mathrm{EE}$ than in groups $\mathrm{HHP}$ and $\mathrm{EH}$. In contrast, mRNA levels of ADIPOR1 were lower $(P<0.05)$ in EE cows than in HHP cows. Transcription levels of RARRES2,CMKLR1 and GPR1 were also higher $(P<0.001, P<0.001$ and $P=0.001$, respectively) in group EE than in groups $\mathrm{HHP}$ and $\mathrm{EH}$, 


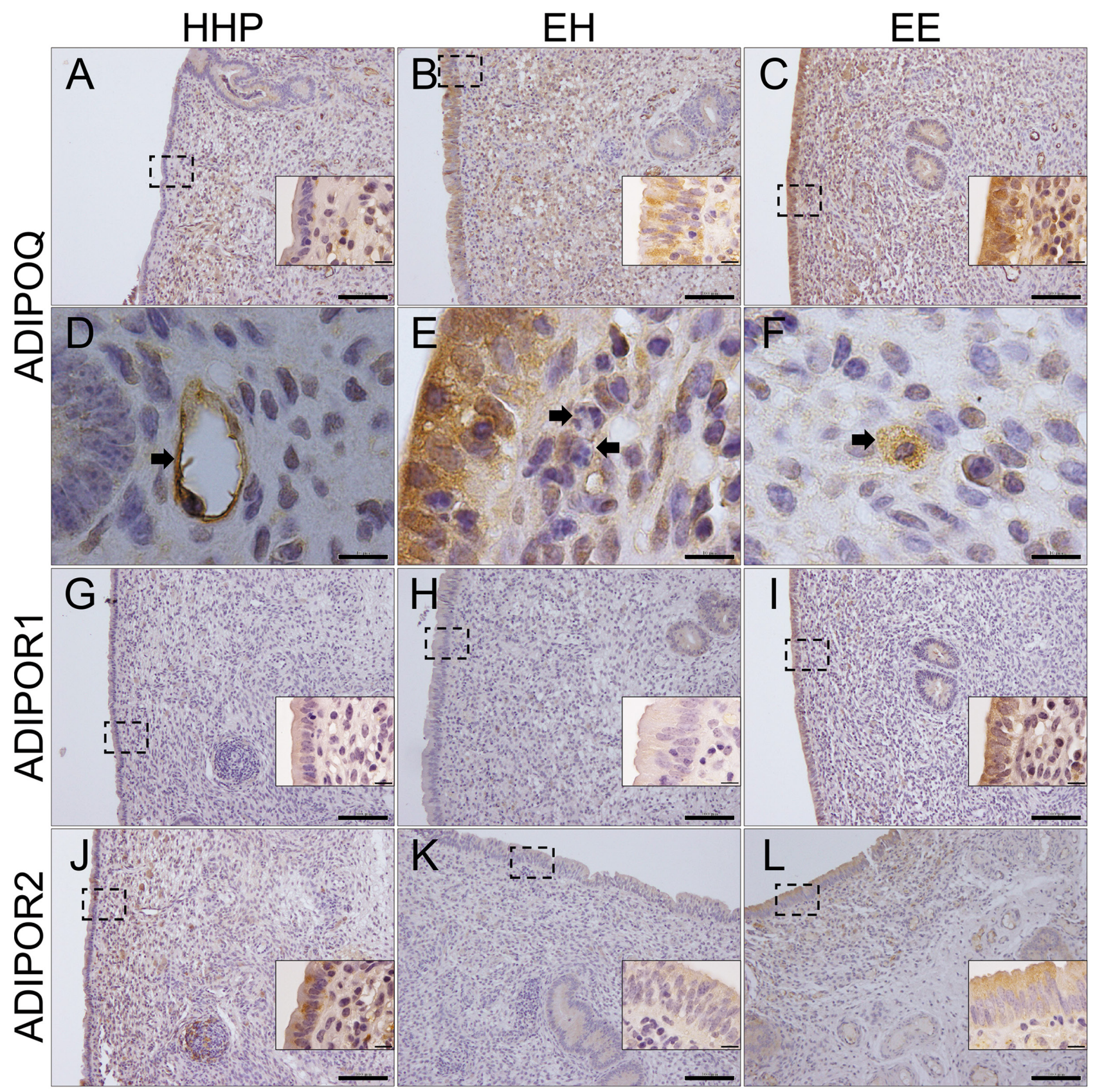

Figure 3 Representative photomicrographs of endometrial sections from biopsies recovered at 45 days postpartum from a subset of healthy cows pregnant at first $\mathrm{Al}$ (group HHP), cows with cytological endometritis at day 21 postpartum but that recovered by day 45 postpartum (group EH), and cows with persistent cytological endometritis until day 45 postpartum (group EE). Immunostaining for ADIPOQ (A, B, C, D, E and F), for ADIPOR1 (G, H and I) and for ADIPOR2 (, $\mathrm{K}$ and $\mathrm{L}$ ). In $\mathrm{D}$, arrow pointing to stained endothelial cell; in $\mathrm{E}$, arrows pointing to non-stained PMN; in $F$, arrow pointing to stained macrophage. Scale bar $10 \mu \mathrm{m}(\mathrm{D}, \mathrm{E}, \mathrm{F}$ and all the insets) and $100 \mu \mathrm{m}(\mathrm{A}, \mathrm{B}, \mathrm{C}, \mathrm{G}, \mathrm{H}, \mathrm{I}, \mathrm{J}, \mathrm{K}$ and L).

whereas transcription levels of CCRL2 were similar in the three groups (data not shown).

\section{Discussion}

There is a wide range in the reported prevalence of cytological endometritis in dairy cows, but it is frequently pointed around $30-35 \%$ between 4 to 9 weeks postpartum (LeBlanc 2008). Here, in cows without puerperal disease, the prevalence of cytological endometritis was $61 \%$ (30 out of 49 ) at 21 DPP and $22 \%$ (11 out of 49 ) at 45 DPP. This shows that over $60 \%$ of early affected cows spontaneously recovered by the end of the voluntary waiting period exhibiting transient 
Table 3 Level of immunostaining of ADIPOQ, ADIPOR1, ADIPOR2, RARRES2 and CMKLR1 in different cell populations of endometrial biopsies taken at 45 DPP in healthy cows (group HHP, $n=3$ ), cows with cytological endometritis at 21 DPP but that recovered by 45 DPP (group EH, $n=3$ ), and cows with persistent cytological endometritis until $45 \mathrm{DPP}$ (group EE, $n=3$ ).

\begin{tabular}{|c|c|c|c|}
\hline & HHP & EH & $\mathrm{EE}$ \\
\hline \multicolumn{4}{|l|}{ ADIPOQ } \\
\hline $\mathrm{LE}$ & $+/-$ & + & ++ \\
\hline GE & + & + & + \\
\hline ST & + & + & + \\
\hline ST-IC & $+/-$ & + & + \\
\hline ST-LF & - & - & $+/-$ \\
\hline \multicolumn{4}{|c|}{ ADIPOR1 } \\
\hline LE & $+/-$ & + & ++ \\
\hline GE & + & + & + \\
\hline ST & - & - & - \\
\hline ST-IC & - & - & - \\
\hline ST-LF & - & - & - \\
\hline \multicolumn{4}{|c|}{ ADIPOR2 } \\
\hline LE & $+/-$ & + & ++ \\
\hline GE & + & + & + \\
\hline ST & + & + & + \\
\hline ST-IC & + & + & + \\
\hline ST-LF & + & $+/-$ & + \\
\hline \multicolumn{4}{|c|}{ RARRES2 } \\
\hline LE & + & + & ++ \\
\hline GE & + & $+/-$ & + \\
\hline ST & + & + & + \\
\hline ST-IC & + & + & + \\
\hline ST-LF & + & $+/-$ & $+/-$ \\
\hline \multicolumn{4}{|l|}{ CMKLR1 } \\
\hline LE & + & + & ++ \\
\hline GE & - & - & - \\
\hline ST & $+/-$ & $+/-$ & + \\
\hline ST-IC & $+/-$ & $+/-$ & ++ \\
\hline ST-LF & $+/-$ & - & $+/-$ \\
\hline
\end{tabular}

DPP, days postpartum; GE, glandular epithelium; LE, luminal epithelium; level of staining: - (absent), +/- (weak), +(moderate), ++ (strong); ST, stroma cells; ST-IC, inflammatory cells in stroma; ST-LF, lymphoid follicles in stroma.

endometritis. This also shows that, from the total number of cows initially recruited at 21 DPP, $22 \%$ still suffer from persistent endometrial inflammation at 45DPP.

At 21 DPP, $83 \%$ of cows with cytological endometritis showed a positive vaginal discharge Metricheck score, indicating the subclinical nature of endometrial inflammation in about $20 \%$ of cows. In contrast, at 45 DPP, only $9 \%$ of cows with cytological endometritis showed a positive vaginal discharge Metricheck score, indicating the subclinical nature of endometritis in about $90 \%$ of cows. These figures, as reported by McDougall et al. (2011), demonstrate a moderate level of agreement between the vaginal discharge Metricheck score and the endometrial cytology PMN percentage at 21 DPP and no agreement at 45 DPP. This may be partially explained by or may reflect the presence of cervicitis or vaginitis instead of endometritis (Westermann et al. 2010). By contrast, the onset of postpartum ovarian activity promotes cervix closure under the influence of progesterone, which may block the flux of uterine contents into the vagina.

Therefore, for descriptive purposes, herein cows with cytological endometritis until 45 DPP are also referred as persistent (cytological) endometritis cows, whereas cows that recovered of cytological endometritis by 45 DPP are also referred as transient (cytological) endometritis cows.

Milk yield, body weight loss and blood NEFA concentrations were not associated to persistent or transient cytological endometritis. These findings are in agreement with Dubuc et al. (2011) and reinforce the subclinical nature of this condition. In dairy cows, plasma concentrations of adipokines are affected by negative energy balance status (Mellouk et al. 2017), variations in white adipose tissue quantities (Reverchon et al. 2014) and milk yield (Kafi et al. 2015). However, no differences were observed here between uterine health groups regarding these parameters. Therefore, it is unlikely that they have contributed to the observed between-group differences in adipokines' gene transcription, protein expression and production described subsequently.

This study evidenced that cows affected by persistent endometritis exhibited increased concentrations of ADIPOQ at 21 and 45 DPP in plasma and at 45 DPP in uterine fluid, compared to healthy and transient endometritis cows. The ROC curve analysis showed that the chance of discriminating a healthy from a persistent endometritis cow, at 45 DPP, was $87 \%$ and $98 \%$ based on plasma concentrations at 21 and 45 DPP, respectively, and $100 \%$ based on uterine fluid concentrations at 45 DPP. In addition, the OR analysis showed that cows with concentrations above the cutoff were a minimum of 3.5 (plasma 21 DPP), 20.4 (plasma 45 DPP) and 33.3 (uterine fluid $45 \mathrm{DPP}$ ) times more at risk of evidencing persistent endometritis at 45 DPP than healthy cows. These observations evidence that ADIPOQ is a promising biomarker, with putative high discriminatory power for the identification of persistent endometritis cows at 45 DPP. In contrast with these results, Kasimanickam et al. (2013), although reporting increased concentrations of serum ADIPOQ in cows affected by metritis and clinical endometritis, found no differences between cows affected by subclinical endometritis and healthy cows. However, the study (Kasimanickam et al. 2013) only included six subclinical endometritis cows, and four cows of the healthy cow group $(n=13)$ were subsequently found to develop clinical endometritis 2 weeks later. Therefore, the inconsistency between the two studies may be explained by differences in population size and categorization of the healthy cow group.

The increased plasma and uterine fluid ADIPOQ concentrations observed in endometritis cows can result from white adipose tissue production or it can originate from local expression in the reproductive tissues, which would indicate, besides an endocrine function, also autocrine or paracrine effects (Reverchon 


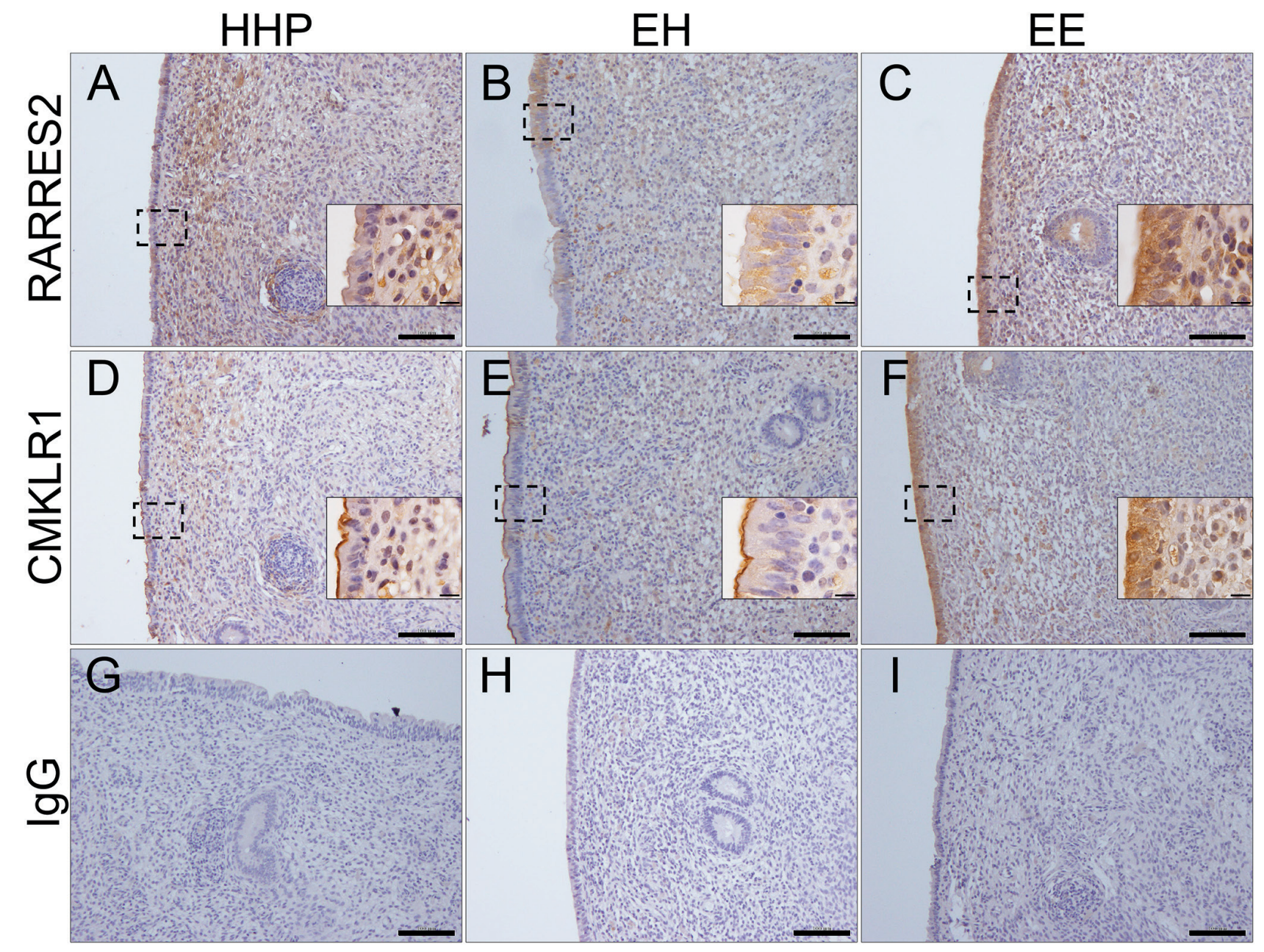

Figure 4 Representative photomicrographs of endometrial sections from biopsies recovered at 45 days postpartum from a subset of healthy cows pregnant at first $\mathrm{Al}$ (group HHP), cows with cytological endometritis at day 21 postpartum but that recovered by day 45 postpartum (group EH), and cows with persistent cytological endometritis until day 45 postpartum (group EE). Immunostaining for RARRES2 (A, B, C), CMKLR1 (D, E, F) and negative control (G, H, I). Scale bar $10 \mu \mathrm{m}$ (insets) and $100 \mu \mathrm{m}(\mathrm{A}, \mathrm{B}, \mathrm{C}, \mathrm{D}, \mathrm{E}, \mathrm{F}, \mathrm{G}, \mathrm{H}$ and I).
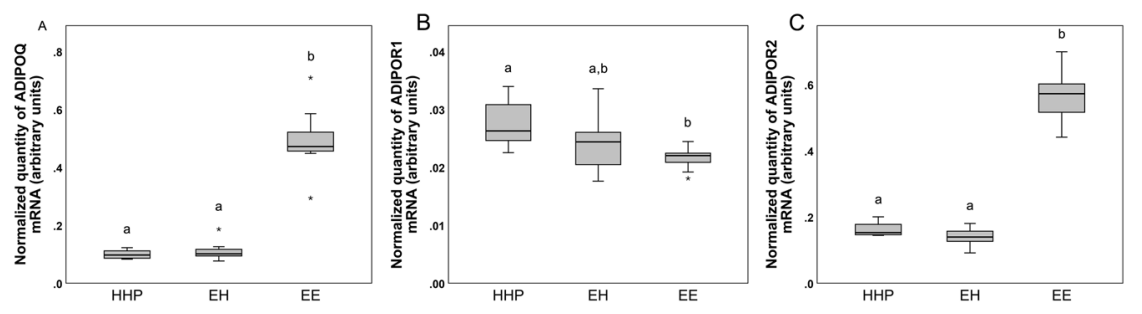

Figure 5 Transcript abundance of $A D I P O Q(\mathrm{~A})$, $A D I P O R 1$ (B) and ADIPOR2 (C) standardized to the geometric mean of GAPDH, ACTB and PPIA in uterine cell pellets collected at 45 days postpartum. Data analysed using Kruskal-Wallis test with Dunns post-test. Different letters indicate a significant difference at $P<0.05$. Groups HHP, healthy cows pregnant at first $\mathrm{Al}$; $\mathrm{EH}$, cows with cytological endometritis at 21 days postpartum but that recovered by 45 days postpartum; EE, cows with persistent cytological endometritis until 45 days postpartum. Horizontal black lines indicate median, boxes extend from the 25th to the 75th percentile and vertical lines indicate values within 1.5 interquartile range of the 25 th and 75 th percentile. Asterisks indicate outliers. 

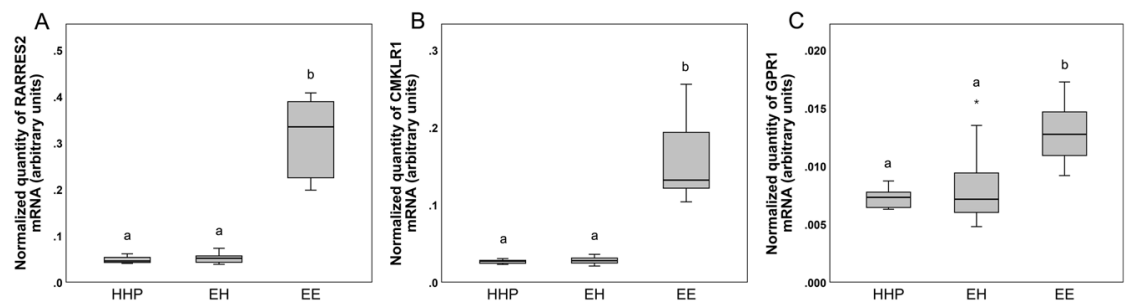

Figure 6 Transcript abundance of RARRES2 (A), CMKLR1 (B) and GPR1 (C) standardized to the geometric mean of GAPDH, ACTB and PPIA in uterine cell pellets collected at 45 days postpartum. Data analysed using Kruskal-Wallis test with Dunns post-test. Different letters indicate a significant difference at $P<0.05$. Groups HHP, healthy cows pregnant at first $\mathrm{Al}$; $\mathrm{EH}$, cows with cytological endometritis at 21 days postpartum but that recovered by 45 days postpartum; $E$, cows with persistent cytological endometritis until 45 days postpartum. Horizontal black lines indicate median, boxes extend from the 25th to the 75th percentile and vertical lines indicate values within 1.5 interquartile range of the 25th and 75 th percentile. Asterisks indicate outliers.

et al. 2014). In this study, the putative uterine origin of ADIPOQ was addressed through the evaluation of gene transcription in the cellular pellet of uterine fluid and the endometrial protein immunolocalisation. Transcription of $A D I P O Q, A D I P O R 1$ and ADIPOR2 was detected in the cellular pellet of uterine flushings, and persistent endometritis increased local transcription of $A D I P O Q$ and its receptors. This may indicate that the presence of ADIPOQ in the uterine lumen is at least partially explained by local transcription. These findings were confirmed by the immunolocalisation of ADIPOQ, ADIPOR1 and ADIPOR2 in the cow endometrium and by the increased immunostaining of these proteins in persistent endometritis cows. Overall, our data indicate that the ADIPOQ signalling system is present in all types of endometrial cells and is related to the inflammatory status of the uterus.

A relationship between ADIPOQ concentrations and the inflammatory status was also observed in the mammary gland of cows following a lipopolysaccharide (LPS) challenge (Singh 2014) and in human patients with inflammatory and immune-mediated diseases (Fantuzzi 2013). ADIPOQ may express anti-inflammatory properties by inducing the switch of macrophage phenotype to an anti-inflammatory state, decreasing the expression of Toll-like receptor 4 (TLR4) and by suppressing the endothelial inflammatory response (Fang \& Judd 2018). The cow innate immune response to LPS is dependent on TLR4 signalling in the epithelial and stromal cells of the endometrium (Herath et al. 2006, Sheldon \& Roberts 2010, Cronin et al. 2012, Piras et al. 2017). In humans, increased TLR4 expression levels were associated with disease progression in patients with chronic endometritis (Ju et al. 2014), and in the bitch, pyometra caused by Escherichia coli, lead to a significantly increased TLR4 transcription and protein expression in all endometrial compartments, including inflammatory cells (Silva et al. 2010). Therefore, endometrial cis and/or trans ADIPOQ signalling may be up-regulated following LPS injury, which may convey early predictive information regarding the establishment of endometritis. Expression of ADIPOQ and its receptors in endometrial endothelial cells is also increased in persistent endometritis cows. Therefore, the antiinflammatory action of ADIPOQ may be also related with the suppression of the endothelial inflammatory response (Fang \& Judd 2018).

This study also evidenced that cows with persistent endometritis exhibited higher plasma and uterine fluid concentrations of RARRES2 at 45 DPP than healthy and transient endometritis cows. Consistent with this, cows with persistent endometritis showed up-regulated transcription of RARRES2,CMKLR1 and GPR1 in the cellular pellet of uterine fluid and an increased expression of RARRES2 and CMKLR1 in the endometrium. These data indicate a relationship between RARRES2 signalling and the inflammatory status of the postpartum cow uterus and a local production of this mediator. However, unlike ADIPOQ, RARRES2 ROC curve and OR analysis revealed that only uterine fluid RARRES2 concentrations were suitable to discriminate persistent endometritis cows at 45 DPP. In fact, cows with uterine fluid concentrations at 45 DPP above the cutoff were a minimum of 33.3 times more at risk of evidencing endometritis at 45 DPP than healthy cows, and concentrations above the cutoff were able to discriminate all healthy from persistent endometritis cows at 45 DPP. Although adipose tissue and liver are considered the major sites of RARRES2 expression, its production has been detected in other tissues including the reproductive tract (Bongrani etal. 2019). This protein is expressed in several epithelial cell types and participates in the host defence mechanisms, as a broad-spectrum antimicrobial protein and as a leukocyte attractant, evidencing pro-inflammatory properties (Zabel et al. 2014). In fact, local RARRES2 concentrations were positively associated with metabolic and inflammatory diseases (Dranse et al. 2015). A systemic repercussion of a local inflammatory status, as evidenced in this study, is consistent with data from Weigert et al. (2010) showing 
increased levels of serum RARRES2 in human patients affected by inflammatory conditions.

Chemerin (RARRES2) is secreted as a precursor termed prochemerin, which is converted into the active form under conditions such as coagulation, fibrinolysis, inflammatory and complement cascades activation (Zabel et al. 2014). These conditions are present in the uterine lumen of cows affected with endometritis, due to the presence of PMNs. These inflammatory cells are the first to be recruited and once activated degranulate and unleash a set of proteases capable of activating prochemerin into RARRES2 (Mariani \& Roncucci 2015). The observed significant positive correlation between RARRES2 concentrations in the uterine fluid, and the endometrial cytology PMN percentage at 45 DPP, is in accordance with the previously mentioned mechanism. Also, the lack of correlation between plasma and uterine concentrations of RARRES2 at 45 DPP is in accordance with a local activation and inactivation of RARRES2 by proteolytic processes (Bondue et al. 2011). The immunolocalisation of RARRES2 in the endometrial luminal and glandular epithelium as well as in the stroma and inflammatory cells lead to the suggestion that RARRES2 also act in the cow endometrium as a player of the pro-inflammatory cascade.

Its receptor CMKLR1 is expressed in the luminal epithelium, either at the apical membrane (groups HHP and $\mathrm{EH}$ ) or throughout the cytoplasm (group EE). As observed in other scenarios (Zhou et al. 2014, De Henau et al. 2016), this may reflect the receptor's internalisation after binding with RARRES2, which appeared here more pronounced in cows with persistent endometritis than in other groups. Plasma and uterine fluid NAMPT concentrations were similar in healthy and persistent endometritis cows. This finding was unexpected since NAMPT was suggested as an indicator of inflammatory disease in cows (Fadden \& Bobe 2016) and to be involved in the modulation of the uterine LPS-induced inflammatory response in rats (Yang et al. 2015).

A significant positive correlation between ADIPOQ and RARRES2 concentrations in the uterine fluid was observed. This may represent the two faces of a balanced inflammatory response, combining anti-inflammatory (ADIPOQ) and pro-inflammatory (RARRES2) characteristics. This could act through a mechanism by which ADIPOQ could induce CMKLR1 expression and, consequently, activation of the RARRES2/CMKLR1 system, as proposed in humans following reduction of weight (Shin et al. 2017).

\section{Conclusions}

In conclusion, postpartum dairy cows affected with persistent endometritis presented increased plasma and uterine fluid concentrations of ADIPOQ and RARRES2, up-regulation of transcription of $A D I P O Q, A D I P O R 1$, ADIPOR2, RARRES2, CMKLR1 and GPR1 in the cellular pellet of uterine fluid, and increased expression of ADIPOQ, ADIPOR1, ADIPOR2, RARRES2 and CMKLR1 in the endometrium. Plasma and uterine fluid ADIPOQ and RARRES2 concentrations were positively correlated with the endometrial cytology PMN percentage, and the ROC curve and OR analysis showed that both plasma and uterine fluid ADIPOQ concentrations and uterine fluid RARRES2 concentrations were able to identify a persistent endometrial inflammation at 45 DPP. These data indicate a relationship between adipokine signalling and the inflammatory status of the postpartum uterus of dairy cows, where ADIPOQ and RARRES2 systems are potentially involved in a balanced anti-inflammatory and pro-inflammatory response, respectively. Therefore, ADIPOQ and RARRES2 represent suitable biomarkers able to provide an early diagnosis of subclinical endometritis and predict the risk of persistence of uterine inflammation. This would be of particular interest for discriminating cows elective for timely therapeutic approaches, as affected cows could be identified early in the postpartum period and allocated to appropriate therapy. Further studies are necessary to determine the role of these adipokines in the establishment of subclinical endometritis.

\section{Supplementary materials}

This is linked to the online version of the paper at https://doi. org/10.1530/REP-20-0183.

\section{Declaration of interest}

Joëlle Dupont is on the editorial board of Reproduction. Joëlle Dupont was not involved in the review or editorial process for this paper, on which she is listed as an author. The other authors have nothing to disclose.

\section{Funding}

Gonçalo Pereira is a PhD student supported by a grant from Fundação para a Ciência e Tecnologia (FCT) (SFRH/ BD/130,923/2017). Elisabete Silva is funded by FCT (DL 57/2016/CP1438/CT0001). This work was supported by FCT (Project UIDP/CVT/00,276/2020) and FORMAS (Grant No 2015-00,888).

\section{Author contribution statement}

G P, P H and L L-d-C designed the study. G P, R B, J C S and L $\mathrm{L}-\mathrm{d}-\mathrm{C}$ conducted clinical evaluations and collected samples. G P, E S, C R and J D performed lab assays (analytical, ELISA, qPCR and IHC assays). Y G participated in the IHC study. G P, E S, J $\mathrm{D}, \mathrm{PH}$ and $\mathrm{L} \mathrm{L}-\mathrm{d}-\mathrm{C}$ participated in data analysis and manuscript preparation. All authors read and approved the final manuscript. $\mathrm{PH}$ and $\mathrm{L} \mathrm{L-d}-\mathrm{C}$ are the shared senior authors. 


\section{Acknowledgements}

The authors thank Barão and Barão Lda for providing facilities for this study.

\section{References}

Adnane M, Chapwanya A, Kaidi R, Meade KG \& O'Farrelly C 2017 Profiling inflammatory biomarkers in cervico-vaginal mucus (CVM) postpartum: potential early indicators of bovine clinical endometritis? Theriogenology 103 117-122. (https://doi.org/10.1016/j.theriogenology. 2017.07.039)

Astessiano AL, Carriquiry M, Mattiauda DA, Adrien ML, Chilibroste P \& Meikle A 2017 Endometrial gene expression in primiparous dairy cows at the end of the voluntary waiting period is affected by nutrition: total mixed ration vs increasing levels of herbage allowance. Reproduction in Domestic Animals 52 798-805. (https://doi.org/10.1111/rda.12981)

Barbe A, Bongrani A, Mellouk N, Estienne A, Kurowska P, Grandhaye J, Elfassy Y, Levy R, Rak A, Froment P et al. 2019 Mechanisms of adiponectin action in fertility: an overview from gametogenesis to gestation in humans and animal models in normal and pathological conditions. International Journal of Molecular Sciences 201526. (https:// doi.org/10.3390/ijms20071526)

Bondue B, Wittamer V \& Parmentier M 2011 Chemerin and its receptors in leukocyte trafficking, inflammation and metabolism. Cytokine and Growth Factor Reviews 22 331-338. (https://doi.org/10.1016/j. cytogfr.2011.11.004)

Bongrani A, Mellouk N, Rame C, Cornuau M, Guérif F, Froment P \& Dupont J 2019 Ovarian expression of adipokines in polycystic ovary syndrome: a role for chemerin, omentin, and apelin in follicular growth arrest and ovulatory dysfunction? International Journal of Molecular Sciences 20 3778. (https://doi.org/10.3390/ijms20153778)

Britt JH, Cushman RA, Dechow CD, Dobson H, Humblot P, Hutjens MF, Jones GA, Ruegg PS, Sheldon IM \& Stevenson JS 2018 Invited review: learning from the future-A vision for dairy farms and cows in 2067. Journal of Dairy Science 101 3722-3741. (https://doi.org/10.3168/ jds.2017-14025)

Chapwanya A, Meade KG, Doherty ML, Callanan JJ, Mee JF \& O'Farrelly C 2009 Histopathological and molecular evaluation of Holstein-Friesian cows postpartum: toward an improved understanding of uterine innate immunity. Theriogenology 71 1396-1407. (https://doi.org/10.1016/j. theriogenology.2009.01.006)

Chapwanya A, Meade KG, Narciandi F, Stanley P, Mee JF, Doherty ML, Callanan JJ \& O'Farrelly C 2010 Endometrial biopsy: a valuable clinical and research tool in bovine reproduction. Theriogenology 73 988-994. (https://doi.org/10.1016/j.theriogenology.2009.11.015)

Cronin JG, Turner ML, Goetze L, Bryant CE \& Sheldon IM 2012 Toll-like receptor 4 and MYD88-dependent signaling mechanisms of the innate immune system are essential for the response to lipopolysaccharide by epithelial and stromal cells of the bovine endometrium. Biology of Reproduction 86 51. (https://doi.org/10.1095/biolreprod.111.092718)

De Henau O, Degroot GN, Imbault V, Robert V, De Poorter C, Mcheik S, Galés C, Parmentier M \& Springael JY 2016 Signaling properties of chemerin receptors CMKLR1, GPR1 and CCRL2. PLOS ONE 11 e0164179. (https://doi.org/10.1371/journal.pone.0164179)

Dranse HJ, Rourke JL, Stadnyk AW \& Sinal CJ 2015 Local chemerin levels are positively associated with DSS-induced colitis but constitutive loss of CMKLR1 does not protect against development of colitis. Physiological Reports 3 e12497. (https://doi.org/10.14814/phy2.12497)

Dubuc J, Duffield TF, Leslie KE, Walton JS \& Leblanc SJ 2011 Effects of postpartum uterine diseases on milk production and culling in dairy cows. Journal of Dairy Science 94 1339-1346. (https://doi.org/10.3168/ jds.2010-3758)

Dupont J, Pollet-Villard X, Reverchon M, Mellouk N \& Levy R 2015 Adipokines in human reproduction. Hormone Molecular Biology and Clinical Investigation 24 11-24. (https://doi.org/10.1515/ hmbci-2015-0034)

Fadden AN \& Bobe G 2016 Serum visfatin is a predictive indicator of retained placenta and other diseases in dairy cows. Journal of Veterinary Science and Medical Diagnosis 5 1. (https://doi.org/10.4172/23259590.1000188)
Fang H \& Judd RL 2018 Adiponectin regulation and function. Comprehensive Physiology 8 1031-1063. (https://doi.org/10.1002/cphy. c170046)

Fantuzzi G 2013 Adiponectin in inflammatory and immune-mediated diseases. Cytokine 64 1-10. (https://doi.org/10.1016/j.cyto.2013.06.317)

Gilbert RO, Shin ST, Guard CL, Erb HN \& Frajblat M 2005 Prevalence of endometritis and its effects on reproductive performance of dairy cows. Theriogenology 64 1879-1888. (https://doi.org/10.1016/j. theriogenology.2005.04.022)

Guo Y, Chankeaw W, Chanrot M, Valarcher JF, Chantarapratep P, Bage R, Bongcam-Rudloff E, Andersson G, Charpigny G \& Humblot P 2019 Changes induced by pathogens and metabolic stress on endometrial function in cattle: possible impacts of increased inflammation on fertility. Revista Brasileira de Reprodução Animal 43 295-307.

Hammon DS, Evjen IM, Dhiman TR, Goff JP \& Walters JL 2006 Neutrophil function and energy status in Holstein cows with uterine health disorders. Veterinary Immunology and Immunopathology 113 21-29. (https://doi. org/10.1016/j.vetimm.2006.03.022)

Herath S, Fischer DP, Werling D, Williams EJ, Lilly ST, Dobson H, Bryant CE \& Sheldon IM 2006 Expression and function of toll-like receptor 4 in the endometrial cells of the uterus. Endocrinology 147 562-570. (https://doi. org/10.1210/en.2005-1113)

Inchaisri C, Jorritsma R, Vos PL, van der Weijden GC \& Hogeveen H 2010 Economic consequences of reproductive performance in dairy cattle. Theriogenology 74 835-846. (https://doi.org/10.1016/j. theriogenology.2010.04.008)

Ju J, Li L, Xie J, Wu Y, Wu X \& Li W 2014 Tolllike receptor4 pathway is required for the pathogenesis of human chronic endometritis. Experimental and Therapeutic Medicine 8 1896-1900. (https://doi. org/10.3892/etm.2014.1990)

Kafi M, Tamadon A \& Saeb M 2015 The relationship between serum adiponectin and postpartum luteal activity in high-producing dairy cows. Theriogenology 83 1264-1271. (https://doi.org/10.1016/j. theriogenology.2015.01.011)

Kasimanickam R, Duffield TF, Foster RA, Gartley CJ, Leslie KE, Walton JS \& Johnson WH 2004 Endometrial cytology and ultrasonography for the detection of subclinical endometritis in postpartum dairy cows. Theriogenology 62 9-23. (https://doi.org/10.1016/j.theriogenology. 2003.03.001)

Kasimanickam RK, Kasimanickam VR, Olsen JR, Jeffress EJ, Moore DA \& Kastelic JP 2013 Associations among serum pro- and anti-inflammatory cytokines, metabolic mediators, body condition, and uterine disease in postpartum dairy cows. Reproductive Biology and Endocrinology 11 103. (https://doi.org/10.1186/1477-7827-11-103)

Kurowska P, Mlyczyńska E, Barbe A, Mellouk N, Dupont J \& Rak A 2018 The adipokines in domestic animal reproduction: expression and role in the regulation of ovarian function. In New Insights into Theriogenology. Ed R Payan-Carreira. London: IntechOpen. (https://doi.org/10.5772/ intechopen.81035)

LeBlanc SJ 2008 Postpartum uterine disease and dairy herd reproductive performance: a review. Veterinary Journal 176 102-114. (https://doi. org/10.1016/j.tvjl.2007.12.019)

Maillard V, Uzbekova S, Guignot F, Perreau C, Ramé C, CoyralCastel S \& Dupont J 2010 Effect of adiponectin on bovine granulosa cell steroidogenesis, oocyte maturation and embryo development. Reproductive Biology and Endocrinology 8 23. (https://doi. org/10.1186/1477-7827-8-23)

Mancuso P 2016 The role of adipokines in chronic inflammation. ImmunoTargets and Therapy 5 47-56. (https://doi.org/10.2147/ITT. S73223)

Mariani F \& Roncucci L 2015 Chemerin/chemR23 axis in inflammation onset and resolution. Inflammation Research 64 85-95. (https://doi. org/10.1007/s00011-014-0792-7)

Mateus L, da Costa LL, Bernardo F \& Silva JR 2002 Influence of puerperal uterine infection on uterine involution and postpartum ovarian activity in dairy cows. Reproduction in Domestic Animals 37 31-35. (https://doi. org/10.1046/j.1439-0531.2002.00317.x)

Mattern A, Zellmann T \& Beck-Sickinger AG 2014 Processing, signaling, and physiological function of chemerin. IUBMB Life 66 19-26. (https:// doi.org/10.1002/iub.1242)

Mayasari N, Chen J, Ferrari A, Bruckmaier RM, Kemp B, Parmentier HK, van Knegsel ATM \& Trevisi E 2017 Effects of dry period length and 
dietary energy source on inflammatory biomarkers and oxidative stress in dairy cows. Journal of Dairy Science 100 4961-4975. (https://doi. org/10.3168/jds.2016-11857)

McDougall S, Hussein H, Aberdein D, Buckle K, Roche J, Burke C, Mitchell M \& Meier S 2011 Relationships between cytology, bacteriology and vaginal discharge scores and reproductive performance in dairy cattle. Theriogenology 76 229-240. (https://doi.org/10.1016/j. theriogenology.2010.12.024)

Mellouk N, Rame C, Touzé JL, Briant E, Ma L, Guillaume D, Lomet D, Caraty A, Ntallaris T, Humblot P et al. 2017 Involvement of plasma adipokines in metabolic and reproductive parameters in Holstein dairy cows fed with diets with differing energy levels. Journal of Dairy Science 100 8518-8533. (https://doi.org/10.3168/jds.2017-12657)

Mellouk N, Ramé C, Diot M, Briant E, Touzé JL, Guillaume D, Froment P \& Dupont J 2019 Possible involvement of the RARRES2/CMKLR1system in metabolic and reproductive parameters in Holstein dairy cows. Reproductive Biology and Endocrinology 17 25. (https://doi. org/10.1186/s12958-019-0467-x)

Parker-Duffen JL, Nakamura K, Silver M, Zuriaga MA, MacLauchlan S, Aprahamian TR \& Walsh K 2014 Divergent roles for adiponectin receptor 1 (AdipoR1) and AdipoR2 in mediating revascularization and metabolic dysfunction in vivo. Journal of Biological Chemistry 289 16200-16213. (https://doi.org/10.1074/jbc.M114.548115)

Pascottini OB, Hostens M, Dini P, Vandepitte J, Ducatelle R \& Opsomer G 2016 Comparison between cytology and histopathology to evaluate subclinical endometritis in dairy cows. Theriogenology 86 1550-1556. (https://doi.org/10.1016/j.theriogenology.2016.05.014)

Piras C, Guo Y, Soggiu A, Chanrot M, Greco V, Urbani A, Charpigny G, Bonizzi L, Roncada P \& Humblot P 2017 Changes in protein expression profiles in bovine endometrial epithelial cells exposed to E. coli LPS challenge. Molecular Biosystems 13 392-405. (https://doi.org/10.1039/ c6mb00723f)

Raliou M, Dembélé D, Düvel A, Bolifraud P, Aubert J, Mary-Huard T, Rocha D, Piumi F, Mockly S, Heppelmann M et al. 2019 Subclinical endometritis in dairy cattle is associated with distinct mRNA expression patterns in blood and endometrium. PLOS ONE 14 e0220244 (https:// doi.org/10.1371/journal.pone.0220244)

Reverchon M, Ramé C, Bertoldo M \& Dupont J 2014 Adipokines and the female reproductive tract. International Journal of Endocrinology $\mathbf{2 0 1 4}$ 232454. (https://doi.org/10.1155/2014/232454)

Rourke JL, Dranse HJ \& Sinal CJ 2013 Towards an integrative approach to understanding the role of chemerin in human health and disease. Obesity Reviews 14 245-262. (https://doi.org/10.1111/obr.12009)

Sheldon IM \& Roberts MH 2010 Toll-like receptor 4 mediates the response of epithelial and stromal cells to lipopolysaccharide in the endometrium. PLOS ONE 5 e12906. (https://doi.org/10.1371/journal.pone.0012906)

Sheldon IM, Lewis GS, LeBlanc S \& Gilbert RO 2006 Defining postpartum uterine disease in cattle. Theriogenology 65 1516-1530. (https://doi. org/10.1016/j.theriogenology.2005.08.021)

Sheldon IM, Cronin JG \& Bromfield JJ 2018 Tolerance and innate immunity shape the development of postpartum uterine disease and the impact of endometritis in dairy cattle. Annual Review of Animal Biosciences 15 361-384. (https://doi.org/10.1146/annurev-animal-020518-115227)

Shin HY, Park S \& Lee JW 2017 Positive association between the changes in chemerin and adiponectin levels after weight reduction. Endocrine Research 42 287-295. (https://doi.org/10.1080/07435800.2 $017.1300808)$

Silva E, Leitão S, Henriques S, Kowalewski MP, Hoffmann B, FerreiraDias G, da Costa LL \& Mateus L 2010 Gene transcription of TLR2, TLR4, LPS ligands and prostaglandin synthesis enzymes are upregulated in canine uteri with cystic endometrial hyperplasia-pyometra complex. Journal of Reproductive Immunology 84 66-74. (https://doi. org/10.1016/j.jri.2009.10.004)
Singh SP 2014 Characterization of adiponectin at different physiological states in cattle based on an in-house developed immunological assay for bovine adiponectin. Doctoral thesis, University of Bonn, Bonn. (available at: http://hss.ulb.uni-bonn.de/2014/3490/3490.pdf)

Smolinska N, Dobrzyn K, Maleszka A, Kiezun M, Szeszko K \& Kaminski T 2014 Expression of adiponectin and adiponectin receptors 1 (AdipoR1) and 2 (AdipoR2) in the porcine uterus during the oestrous cycle. Animal Reproduction Science 146 42-54. (https://doi.org/10.1016/j. anireprosci.2014.02.001)

Takemura Y, Osuga Y, Yamauchi T, Kobayashi M, Harada M, Hirata T, Morimoto C, Hirota Y, Yoshino O, Koga K et al. 2006 Expression of adiponectin receptors and its possible implication in the human endometrium. Endocrinology 147 3203-3210. (https://doi.org/10.1210/ en.2005-1510)

Vandesompele J, De Preter K, Pattyn F, Poppe B, Van Roy N, De Paepe A \& Speleman F 2002 Accurate normalization of real-time quantitative RT-PCR data by geometric averaging of multiple internal control genes. Genome Biology 3 RESEARCH0034. (https://doi.org/10.1186/gb-20023-7-research0034)

Weigert J, Obermeier F, Neumeier M, Wanninger J, Filarsky M, Bauer S, Aslanidis C, Rogler G, Ott C, Schäffler A et al. 2010 Circulating levels of chemerin and adiponectin are higher in ulcerative colitis and chemerin is elevated in Crohn's disease. Inflammatory Bowel Diseases 16 630-637. (https://doi.org/10.1002/ibd.21091)

Westermann S, Drillich M, Kaufmann TB, Madoz LV \& Heuwieser W 2010 A clinical approach to determine false positive findings of clinical endometritis by vaginoscopy by the use of uterine bacteriology and cytology in dairy cows. Theriogenology 74 1248-1255. (https://doi. org/10.1016/j.theriogenology.2010.05.028)

Williams EJ, Fischer DP, Pfeiffer DU, England GC, Noakes DE, Dobson H \& Sheldon IM 2005 Clinical evaluation of postpartum vaginal mucus reflects uterine bacterial infection and the immune response in cattle. Theriogenology 63 102-117. (https://doi.org/10.1016/j. theriogenology.2004.03.017)

Yang Z, Xiao K, Wang W, Tang J, Sun PP, Peng KM \& Song H 2015 The effect of visfatin on inflammatory reaction in uterus of LPS-induced rats. International Journal of Morphology 33 194-203. (https://doi. org/10.4067/S0717-95022015000100031)

Yoshimura T \& Oppenheim J 2011 Chemokine-like receptor 1 (CMKLR1) and chemokine (C-C motif) receptor-like 2 (CCRL2); two multifunctional receptors with unusual properties. Experimental Cell Research 317 674-684. (https://doi.org/10.1016/j.yexcr.2010.10.023)

Zabel BA, Kwitniewski M, Banas M, Zabieglo K, Murzyn K \& Cichy J 2014 Chemerin regulation and role in host defense. American Journal of Clinical and Experimental Immunology 3 1-19.

Zhang Y, Huo Y, He W, Liu S, Li H \& Li L 2019 Visfatin is regulated by interleukin6 and affected by the PPAR $\gamma$ pathway in BeWo cells. Molecular Medicine Reports 19 400-406. (https://doi.org/10.3892/ mmr.2018.9671)

Zhou JX, Liao D, Zhang S, Cheng N, He HQ \& Ye RD 2014 Chemerin C9 peptide induces receptor internalization through a clathrin-independent pathway. Acta Pharmacologica Sinica 35 653-663. (https://doi. org/10.1038/aps.2013.198)

Received 1 April 2020

First decision 19 May 2020

Revised manuscript received 12 June 2020

Accepted 18 June 2020 